\title{
Relationship between recovery of motor function and neuropsychological functioning in cerebral infarction patients: the importance of social functioning in motor recovery
}

Min Cheol Chang ${ }^{1}$, Sung-Won Park ${ }^{2}$, Byung-Joo Lee ${ }^{2, t, *}$ and Donghwi Park ${ }^{3, t, *}$

\author{
${ }^{1}$ Department of Rehabilitation Medicine, College of Medicine, Yeoungnam University, Daegu, 42415, Republic of Korea \\ 2 Department of Rehabilitation Medicine, Daegu Fatima Hospital, Daegu, 41 199, Republic of Korea \\ ${ }^{3}$ Department of Physical Medicine and Rehabilitation, Ulsan University Hospital, University of Ulsan College of Medicine, \\ Ulsan, 44033, Republic of Korea
}

*Correspondence: bdome@hanmail.net (Donghwi Park); bj|84@naver.com (Byung-Joo Lee)

${ }^{\dagger}$ These authors contributed equally.

DOI: $10.31083 /$ i.jin.2020.03.175

This is an open access article under the CC BY 4.0 license (https://creativecommons.org/licenses/by/4.0/).

Impaired motor function is a common disabling sequela after stroke. It is closely associated with the patient's quality of life and independence. Neuropsychological dysfunctions also frequently occur in stroke patients. In this paper, we evaluate the relationship between the recovery of motor function and neuropsychological functions, including cognition, language, emotion, behavior, personality, and social interaction, to provide appropriate and effective therapy for stroke patients. Motor function, neuropsychological status, social functioning, as well as emotional aspects such as depression and anxiety symptoms, were initially evaluated one month after cerebral infarction onset. The evaluations were repeated three months after the onset. Motor function was assessed with the Modified Barthel Index. The neuropsychological status was evaluated using the Mini-Mental State Examination, Global Deterioration Scale, digit span test, Korean-Boston Naming Test, Vineland Social Maturity Scale, Neuropsychiatric Inventory, Beck's Depression Inventory, and Beck Anxiety Inventory. In the results, the Modified Barthel Index, MiniMental State Examination, Global Deterioration Scale, digit span test, and Vineland Social Maturity Scale were significantly different between the two-time points $(P<$ 0.05). Initial Social Maturity Scale Social Age and Social Maturity Scale Social Quotient categories of the Vineland Social Maturity Scale and Mini-Mental State Examination scores were significantly correlated with Modified Barthel Index improvement $(P<0.05)$. The amount of change in the Social Maturity Scale Social Age and Social Maturity Scale Social Quotient scores was significantly correlated with Modified Barthel Index improvement $(P<0.05)$. In multiple linear regression analysis, only the initial Social Maturity Scale Social Quotient score and the amount of score change in Social Maturity Scale Social Quotient showed a significant correlation with Modified Barthel Index improvement $(P<0.05)$. Social function and interaction are important in motor recovery of ischemic stroke patients.

\section{Keywords}

Motor dysfunction; cerebral infarction; neuropsychological functioning, motor dysfunction; social functioning; social interaction

\section{Introduction}

Deterioration of motor function frequently occurs in cerebral infarction patients and is closely associated with the quality of life and independence in activities of daily living after stroke (Ones et al., 2005). After a stroke, in addition to motor function, various functions can be affected. For a detailed evaluation of patients' dysfunction, neuropsychological tests are frequently performed in stroke patients. Using these tests, various brain functions involved in cognition, language, emotion, behavior, personality, and social functioning can be evaluated (Verhoeven et al., 2011). Based on the neuropsychological test results, clinicians can obtain detailed information about dysfunction and elucidate an effective treatment strategy (Tyburski et al., 2018).

Recently, some studies evaluated the association between motor recovery and neuropsychological functioning in stroke patients and reported significant correlations between them (D'Anci et al., 2019; Ginex et al., 2017; McDonald et al., 2019; Mead et al., 2020; Mullick et al., 2015; Nannetti et al., 2005; Rubio Ballester et al., 2012). Various neuropsychological functions, such as depression, cognitive function, and social capacity, were reported to be associated with motor recovery after stroke (Parikh et al., 1990; Pohjasvaara et al., 2001). Using meta-analysis, Mullick et al. (2015) reported the associations between cognitive deficits and upper limb motor recovery in studies of patients with stroke. Žikić et al. (2015) also reported that the patients with depression had significantly 
more severe functional disabilities both at baseline and after rehabilitation treatment. However, the potential for functional recovery in depressed patients was less than in non-depressed ones (Žikić et al., 2015). Venna et al. (2014) also reported the role of social factors on recovery after stroke, and the beneficial effects of social interaction on improving stroke recovery.

However, the conclusions remain controversial. Some previous studies reported that post-stroke depression and cognitive function were not influential in motor recovery (Nannetti et al., 2005). These inconsistent results seem to be because stroke onset and location of stroke lesion of participants in the previous studies were heterogeneous. And it can also be attributed to several factors, including sample size, use of different diagnostic criteria, methodological differences in case selection and screening tools, and the time interval between the stroke event and assessment. Moreover, most studies evaluated particular aspects of neuropsychological function, rather than undergoing more comprehensively evaluation (McDonald et al., 2019; Mead et al., 2020; Rubio Ballester et al., 2012).

\section{Material and methods \\ 2.1 Participants}

The institutional review board approved this study of our hospital (DEF20ORIO079). Thirty-seven patients were included in our study. They were clinically diagnosed with a stroke, according to the World Health Organization ${ }^{1}$, and were admitted to the department of rehabilitation medicine in our hospital. Our study was conducted retrospectively. Ischemic stroke patients, in their subacute stage, were included in this study. The inclusion criteria were: (1) patients with an event of ischemic stroke within the past 3 months, and (2) those who completed the Modified Barthel Index (MBI) and psychological evaluation at 1 and 3 months after the stroke. The exclusion criteria were: (1) a history of previous brain injuries, such as stroke or traumatic brain injury, (2) any other neurological disorders that preclude the completion of the MBI and psychological evaluation, (3) a history of depression or other psychiatric disorder, and (4) preliminary MBI and psychological evaluation at the designated time points.

To improve functional and cognition recovery, some medications related to neurotransmitters were being used by the patients. A selective serotonin reuptake inhibitor (Escitalopram, Lexapro®, Lundbeck) was prescribed to all patients who were included in our study, and an acetyl-cholinesterase inhibitor (Donepezil, Aricept ${ }^{\circledR}$, Eisai) was prescribed to 25 patients with Mini-Mental State Examination (MMSE) score lower than 24. All these medications were prescribed at least one week before the first functional and neuropsychological evaluation.

\subsection{Functional, cognitive, and neuropsychological evaluation}

The participants' motor function and neuropsychological status (neuropsychological and social functioning, as well as emotional aspects such as depression and anxiety symptoms) were initially evaluated 1 month after the stroke onset. The evaluations were

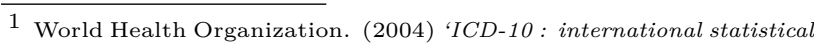
classification of diseases and related health'. Available at: https://ww w.who.int/classifications/icd/en/.
}

repeated 3 months after the onset. Motor function was assessed using the MBI. The cognitive and neuropsychological status was evaluated using the MMSE, Global Deterioration Scale (GDS), Digit Span Test from the Wechsler Adult Intelligence Scale-IV, and Korean-Boston Naming Test (KBNT). Social functioning, as was assessed using the Vineland Social Maturity Scale (VSMS), and the emotional aspects were assessed using the Neuropsychiatric Inventory (NPI), Beck's Depression Inventory (BDI), and Beck Anxiety Inventory (BAI).

\subsubsection{Modified Barthel index}

The MBI is a measure of activities of daily living, which shows the degree of independence of a patient from any assistance (Lee et al., 2020). The MBI consists of 10 categories: 5 points for personal hygiene, 5 points for bathing, 10 points for eating, 10 points for using the toilet, 10 points for climbing stairs, 10 points for wearing clothes, 10 points for defecating, 10 points for urinating, 15 points for walking, and 15 points for using the bed (Shah et al., 1989). The highest possible score is 100 points. A score of $0-24$ points indicates complete dependency in activities of daily life; 25-48, maximum dependency; 50-74, partial dependency; 75-90, slight dependency; 91-99, minimum dependency; and 100, complete independency.

\subsubsection{Mini-mental state examination}

The MMSE is a 30-point screening measure, which is used to measure cognitive function (Tombaugh and McIntyre, 1992). It consists of registration, recall, attention, calculation, language, simple command following, and orientation. Any score higher than 24 indicates a normal cognitive function (Kapur, 2005). Below this, scores may indicate mild (19-23 points), moderate (1018 points), or severe ( $\leq 9$ points) cognitive impairment. The raw score was corrected for age and educational status.

\subsubsection{Global deterioration scale}

The GDS is a clinical rating instrument that is used to assess stages of primary degenerative dementia (Reisberg et al., 1982). It consists of a 7-point scale: no cognitive decline (stage 1), very mild cognitive decline (stage 2), mild cognitive decline (stage 3), moderate cognitive decline (stage 4), moderately severe cognitive decline (stage 5), severe cognitive decline (stage 6), and very severe cognitive decline (stage 7). The measure was completed by the patient and rated by the attending psychiatrist.

\subsubsection{Digit span test forward \& backward}

The Digit Span Test is a subtest of both the Wechsler Adult Intelligence Scale and the Wechsler Memory Scales (Drozdick et al., 2018). Subjects are read a sequence of numbers and asked to repeat the same sequence back to the examiner in order (forward span) or reverse order (backward span). A digit span test is performed to measure the number of storage capacity of working memory (Wilde et al., 2004). It is believed that Digit Span "forward" is a simple repetition task and involves mostly auditory short-term memory. The "backward" variant, on the other hand, is a more complicated task and requires manipulation of information stored in short-term memory, which makes it a better measure of working memory. Subjects see or hear a sequence of numerical digits and are asked to recall the sequence correctly, with longer sequences 
being tested in each trial. The digit span test is administered either forward or backward, which means that once the sequence is presented to the subjects, they are asked to recall the sequence in the normal or reverse order. The subject had two trials for each span. The subject's final score was maximum digits repeated without any error in one of the two trials.

\subsubsection{Korean-Boston naming test}

KBNT is the Korean version of the Boston Naming Test (Kim and Na, 1999). The KBNT contains the same number of test items and uses the identical administration method as the original Boston Naming Test. It is a neuropsychological assessment tool for measuring confrontational word retrieval in individuals with aphasia or other language disorders. This test consists of 60 line drawings of objects of graded difficulty, ranging from very common objects (e.g., a tree) to less familiar objects, such as an abacus.

\subsubsection{Vineland social maturity scal}

The VSMS is a psychometric assessment tool that is designed to help measure the different social capacities of an individual (Sparrow et al., 2005). It is mainly used to assess social and adaptive functions or social competency. It provides an estimate of Social Age (SMSSA) and Social Quotient (SMSSQ). It consists of 8 subscales: communication skills, general self-help ability, locomotion skills, occupation skills, self-direction, self-help eating, self-help dressing, and socialization skills. The scale consists of 89 test items grouped into year levels. Based on the total score, the social age is determined using a decimal table. Finally, using social age and chronological age, the social quotient is calculated by dividing social age by chronological age and multiplying with 100. The measure was completed by the patient and rated by the attending psychiatrist.

\subsubsection{Neuropsychiatric inventory}

The NPI was developed to assess psychopathology in dementia patients (Cummings, 1997). It evaluates 12 neuropsychiatric features commonly observed in dementia: delusions, hallucinations, agitation, dysphoria, anxiety, apathy, irritability, euphoria, disinhibition, aberrant motor behavior, night-time behavior disturbances, and appetite and eating abnormalities. The NPI is administrated to caregivers. A set of screening questions is asked about each sub-domain. Only if the responses to these questions indicate that the patient has problems with a particular sub-domain of behavior, the caregiver is then asked of all the questions about that domain. The frequency of the symptoms is rated on a 4-point scale, their severity on a 3-point scale, and the distress the symptom causes on them on a 5-point scale.

\subsubsection{Beck's depression inventory}

The BDI is a 21-question multiple-choice self-report instrument that assesses the characteristic attitudes and symptoms of depression (Beck, 1961). The questionnaire was developed from clinical observations of attitudes and symptoms frequently occurring in depressed psychiatric patients and infrequently in nondepressed psychiatric patients. The questionnaire is commonly self-administered, although initially designed to be administered by trained interviewers. A value of 0 to 3 is assigned for each answer, and then the total score is calculated to determine the severity
Table 1. Clinical and demographic characteristics of included patients with cerebral infarction

\begin{tabular}{lc}
\hline & Patients \\
\hline Age (years) & $61.92 \pm 16.78$ \\
Sex (M : F) & $20: 17$ \\
Side (Lt : Rt : Bi-lateral) & $20: 13: 04$ \\
& PCA $(\mathrm{n}=2)$ \\
MCA $(\mathrm{n}=11)$ \\
ACA $(\mathrm{n}=4)$ \\
Lesions & Anterior choroidal artery $(\mathrm{n}=5)$ \\
& Basal ganglia $(\mathrm{n}=9)$ \\
& Thalamus $(\mathrm{n}=1)$ \\
& Pontine $(\mathrm{n}=3)$ \\
Medulla $(\mathrm{n}=2)$
\end{tabular}

M : F, male : female; Lt : Rt : Quadri, Left ischemic stroke lesion : right ischemic stroke lesion : Bi-lateral ischemic stroke lesions; PCA, posterior cerebral artery; MCA, middle cerebral artery; ACA, anterior cerebral artery.

of depression. A score of 0-9 indicates minimal depression, 10-18 indicates mild depression, 19-29 indicates moderate depression, and 30-63 indicates severe depression. In our study, the measure was completed by the patient and rated by the attending psychiatrist.

\subsubsection{Beck anxiety inventory}

The BAI is a 21-question multiple-choice self-report instrument that is used for measuring the severity of anxiety in children and adults (Beck et al., 1988). It is designed for individuals who are of 17 years of age or older and take 5 to 10 minutes to complete. Each answer is scored on a scale of 0 (not at all) to 3 (severely), and raw scores ranging from 0 to 63 . Higher total scores indicate more severe anxiety. A score of 0-7 indicates minimal anxiety, 815 indicates mild anxiety, 16-25 indicates moderate anxiety, and 26-63 indicates severe anxiety. The measure was completed by the patient and rated by the attending psychiatrist.

\subsection{Statistical analysis}

The Shapiro-Wilk test was used to determine whether the data conformed to a normal distribution. The differences in functional scale and neuropsychological evaluations between two-time points were analyzed using the Student $t$-test. The correlation between the improvement of MBI scores between two-time points (MBI score at 3 months - MBI score at 1 month) and initial neuropsychological evaluations was analyzed with Spearman correlation or Pearson correlation test according to the distribution of the data. The correlation between the improvement of MBI scores between two-time points (MBI score at 3 months - MBI score at 1 month) and improvement of neuropsychological evaluations between twotime points was also analyzed with Spearman correlation or Pearson correlation test according to the distribution of the data. Using the components in the neuropsychological evaluation that significantly correlated with the results of the MBI, multiple linear regression analysis was performed to identify neuropsychological parameters that significantly affected the improvement of motor function. To identify which variables are affected by multi- 
Table 2. Changes in motor function and neuropsychological status

\begin{tabular}{lcc}
\hline & $1^{s t}$ evaluation & $2^{\text {nd }}$ evaluation \\
\hline MBI & $26.86 \pm 19.87$ & $45.08 \pm 26.99$ \\
MMSE & $17.38 \pm 8.79$ & $18.81 \pm 7.67$ \\
DSTF & $4.18 \pm 1.76$ & $4.67 \pm 1.58$ \\
DSTB & $1.97 \pm 1.98$ & $2.33 \pm 1.74$ \\
KBNT & $20.82 \pm 19.05$ & $26.06 \pm 19.27$ \\
SMSSA & $2.68 \pm 2.41$ & $3.65 \pm 3.26$ \\
SMSSQ & $16.78 \pm 15.03$ & $22.78 \pm 20.33$ \\
NPI & $8.30 \pm 19.02$ & $8.00 \pm 14.74$ \\
BDI & $13.82 \pm 8.73$ & $13.94 \pm 11.67$ \\
BAI & $5.61 \pm 8.04$ & $3.47 \pm 3.935$ \\
GDS & $4.51 \pm 1.69$ & $3.91 \pm 1.65$ \\
\hline
\end{tabular}

MMSE, mini-mental state examination; DSTF, digital span test forward; DSTB, digital span test backward; KBNT, Korean-Boston naming test; SMSSA, the social age category of social maturity scale; SMSSQ, social quotient category of social maturity scale; NPI, neuropsychiatric inventory; BDI, Beck's depression inventory; BAI, Beck anxiety inventory; GDS, global deterioration scale.

collinearity, and the strength of the correlation, testing for multicollinearity with Variance Inflation Factors (VIF) was also performed. Multicollinearity is thought to be present when the VIF is higher than 5 to 10 . Then, the multiple linear regression test was re-performed after removing parameters with multicollinearity. Statistical analyses were performed using SPSS for Windows and R package for Windows (version 2.15.2; R Foundation for Statistical Computing, Vienna, Austria).

\section{Results}

A total of 37 patients with ischemic stroke were included in the study (Table 1). Their mean age was $61.92 \pm 16.78$ years, and the sex ratio (male : female) was $20: 17$. Among them, 32 patients had supratentorial stroke lesions, and 5 patients had infratentorial stroke lesions. Twenty patients had left-side hemiplegia (or hemiparesis), 13 participants had right side hemiplegia (or hemiparesis), and 4 had quadriplegia (or quadriparesis). Their first psychosocial evaluations were performed at $30.87 \pm 5.51$ days after stroke onset, and the interval between first and second psychosocial evaluations was $61.79 \pm 19.29$ days. The results of the first and second neuropsychological evaluation tests are presented in Table 2.

\subsection{Change in functional and neuropsychological evaluations between 1 month and 3 months after stroke onset}

A paired $t$-test showed that MMSE, Digit span test forward \& backward, MBI, SMSSQ, SMSSA, and GDS were significantly improved between 1 month and 3 months after stroke onset $(P<$ $0.05)$. However, BDI, BAI, and NPI were not significantly different between 1 month and 3 months after stroke onset $(P \geq 0.05)$.

\subsection{Relationship between functional improvement and initial neuropsychological assessments}

A simple correlation test showed that initial SMSSA, SMSSQ, and MMSE were significantly correlated with MBI improvement
Table 3. Relationship between functional improvement (change of MBI; $\triangle \mathrm{MBI}$ ) and initial neuropsychological assessments

\begin{tabular}{lcc}
\hline Variables & $r$ & $P$-value \\
\hline MMSE & 0.419 & $<\mathbf{0 . 0 5 *}$ \\
DSTF & 0.132 & 0.465 \\
DSTB & 0.224 & 0.211 \\
KBNT & 0.405 & 0.018 \\
SMSSA & 0.472 & $<\mathbf{0 . 0 5 *}$ \\
SMSSQ & 0.469 & $<\mathbf{0 . 0 5 *}$ \\
NPI & 0.193 & 0.252 \\
BDI & 0.046 & 0.799 \\
BAI & 0.036 & 0.842 \\
GDS & 0.287 & 0.086
\end{tabular}

MMSE, Mini-Mental State Examination; DSTF, Digital Span Test Forward; DSTB, Digital Span Test Backward; KBNT, KoreanBoston Naming Test; SMSSA, Social Age category of Social Maturity Scale; SMSSQ, Social Quotient category of Social Maturity Scale; NPI, Neuropsychiatric Inventory; BDI, Beck's Depression Inventory; BAI, Beck Anxiety Inventory; GDS, Global Deterioration Scale.

(Table 3). In multiple linear regression analysis, only SMSSQ showed a statistically significant correlation with MBI improvement (Table 4). However, in this analysis, SMSSA showed multicollinearity, so it was removed in the multiple linear regression analysis.

\subsection{Relationship between functional improvement and changes in neuropsychological assessments}

A simple correlation test showed that the amount of score change in SMSSA and SMSSQ was significantly correlated with MBI improvement (Table 5). In multiple linear regression analysis, only the amount of score change in SMSSQ showed a statistically significant correlation with MBI improvement (Table 6). However, in this analysis, SMSSA showed multicollinearity, so it was removed in the multiple linear regression analysis.

\section{Discussion}

We evaluated the association between the recovery of motor function and neuropsychological functioning, including cognition, language, emotion, behavior, personality, and social interaction measured by a neuropsychological test. The results show that the SMSSQ score 1 month after stroke onset and improvement in the SMSSQ score from 1 to 3 months after the onset correlated with the improvement in motor function in the same period. However, results of other neuropsychological tests, including MMSE, GDS, digit span test, KBNT, SMSSA, NPI, BDI, and BAI, were not associated with improvement in motor function.

It is suggested that social interaction plays a critical role in the recovery after stroke (Glass et al., 1993; Karelina et al., 2009; Rubio Ballester et al., 2012; Venna et al., 2014). In contrast, social isolation may hinder neural regeneration and recovery of motor function after stroke (Glass et al., 1993). Previous animal studies showed the importance of social interaction in motor recovery after stroke. For example, mice paired with a healthy partner showed 
Table 4. Multiple linear regression analysis for evaluating meaningful initial neuropsychological assessments in predicting functional improvement (change of MBI; $\triangle \mathrm{MBI}$ )

\begin{tabular}{cccccc}
\hline Parameter & $\mathrm{R}^{2}$ & Beta coefficient & Standard error & Odd ratio (95\% CI) & $P$-value \\
\hline SMSSQ & 0.145 & 0.380 & 0.127 & $0.276(0.016-0.535)$ & $\mathbf{0 . 0 3 8}^{*}$ \\
\hline
\end{tabular}

Table 5. Relationship between functional improvement (change of MBI; $\triangle \mathrm{MBI}$ ) and changes in neuropsychological assessments

\begin{tabular}{lcc}
\hline Variables & $r$ & $P$-value \\
\hline$\triangle$ MMSE & 0.11 & 0.516 \\
$\triangle$ DSTF & 0.033 & 0.856 \\
$\triangle$ DSTB & 0.079 & 0.663 \\
$\triangle$ KBNT & 0.105 & 0.56 \\
$\triangle$ SMSSA & 0.609 & $<\mathbf{0 . 0 5}^{*}$ \\
$\triangle$ SMSSQ & 0.601 & $<\mathbf{0 . 0 5 *}$ \\
$\triangle$ NPI & 0.248 & 0.144 \\
$\triangle$ BDI & 0.163 & 0.374 \\
$\triangle$ BAI & 0.329 & 0.066 \\
$\triangle$ GDS & 0.246 & 0.154 \\
\hline
\end{tabular}

MMSE, Mini-Mental State Examination; DSTF, Digital Span Test Forward; DSTB, Digital Span Test Backward; KBNT, Korean-Boston Naming Test; SMSSA, Social Age category of Social Maturity Scale; SMSSQ, Social Quotient category of Social Maturity Scale; NPI, Neuropsychiatric Inventory; BDI, Beck's Depression Inventory; BAI, Beck Anxiety Inventory; GDS, Global Deterioration Scale.

better motor recovery compared to isolated mice (Karelina et al., 2009; Venna et al., 2014). Moreover, the level of brain-derived neurotrophic factors, which have neuroprotective functions such as, neural regeneration, and neurogenesis, was enhanced in mice with a healthy partner (Venna et al., 2014). The SMSSQ score in VSMS reflects social capacities, and a low score means that social interaction with other people is difficult or minimal (Sparrow et al., 2005). We observed that a higher initial SMSSQ score or more considerable improvement in the SMSSQ score was associated with better motor recovery after stroke. Accordingly, social interaction seems to play an important role in improving motor recovery after stroke.

We evaluated other areas, such as cognitive function, language function, neuropsychiatric features, and degrees of depression and anxiety, which were not correlated with motor improvement. Regarding the impact of neuropsychological function on motor recovery, inconsistencies among the results of previous studies exist (D'Anci et al., 2019; Dhand et al., 2019; Ginex et al., 2017; Glymour et al., 2008; McDonald et al., 2019; Mead et al., 2020; Mullick et al., 2015; Nannetti et al., 2005; Robinson et al., 1999). For example, some previous studies showed that the treatment of poststroke depression could improve motor and functional recovery (Chollet et al., 2011; Yi et al., 2010). However, a recently conducted meta-analysis showed that it does not improve recovery in motor function after stroke (Mead et al., 2020). Also, Mullick et al. (2015) reported that executive function and attention improved motor function after stroke, but memory function was not associ- ated with motor outcome.

Regarding human studies on the effect of social functioning or interaction on the recovery of stroke, to the best of our knowledge, to date, two studies have been reported (Glass et al., 1993; Rubio Ballester et al., 2012). Glass et al. (1993) recruited 46 stroke patients and measured motor function with the Barthel Index and the degree of social support, 1, 3, and 6 months after stroke onset. Patients with excellent social support were 68 points (65\%) higher than socially isolated patients. Rubio Ballester et al. (2012) recruited eight stroke patients and applied virtual reality (VR)based rehabilitation. The rehabilitation using VR was conducted in single- and multi-player VR environments. The rehabilitation using a multi-player VR environment-induced social interaction among the players. After applying rehabilitation in the multiplayer VR environment, the patients' motor improvements of the affected upper limbs were more significant than those in the singleplayer VR environment.

Our results emphasize the importance of social function and interaction in motor recovery after stroke. Furthermore, we considered and adjusted for several other neuropsychological areas, such as cognitive function, language function, neuropsychiatric features, and degrees of depression and anxiety. We found that social functioning plays a vital role in better outcomes of motor function after stroke. However, there are a few limitations that may influence this conclusion. Firstly, the tool we used for measuring social functioning, namely, SMSSQ, contains items related to motor function, such as locomotion skills and self-help dressing and eating (Sparrow et al., 2005). Therefore, the results of SMSSQ in this study might not solely reflect our patients' social functioning. The number of included patients was relatively small. Therefore, further study with a large number of patients, using various parameters that can better reflect the patients' social functioning is needed in the future. Secondly, we did not use any tool to assess executive functions, which are often impaired after stroke (Povroznik et al., 2018)-the Reliable Change Index (RCI), which is a more sophisticated method for examining change and often used in neuropsychological studies (Duff, 2012), was not performed.

Thirdly, we did not evaluate the correlation between functional improvement, psychosocial evaluation, type of paresis, and location of the stroke. It has been well reported that the location of the stroke is one of the most important factors that may affect improvement (Alawieh et al., 2018). Many well-designed studies adjusted the location of the stroke for finding decisive factors for motor recovery after stroke. For example, for evaluating the influence of diabetes and neural tract integrity on post-stroke motor recovery, Kim et al. (2020) and Moon et al. (2019) specifically included patients with corona radiata lesion (Kim et al., 2020; Moon et al., 2019). Therefore, a further study evaluating the correlations between functional improvement, psychosocial evaluation, type of paresis, and location of the stroke may be necessary. Fourthly, ini- 
Table 6. Multiple linear regression analysis for evaluating meaningful changes in neuropsychological assessments in predicting functional improvement (change of MBI; $\triangle \mathrm{MBI}$ )

\begin{tabular}{cccccc}
\hline Parameter & $\mathrm{R}^{2}$ & Beta coefficient & Standard error & Odd ratio (95\% CI) & $P$-value \\
\hline SMSSQ & 0.201 & 0.448 & 0.192 & $0.511(0.116-0.905)$ & $\mathbf{0 . 0 1 3}^{*}$ \\
\hline
\end{tabular}

tial degrees of motor dysfunction or executive dysfunction might affect our results on motor recovery (Mullick et al., 2015). Finally, we may have excluded valuable parameters, such as education level, that could have been influenced the conclusion.

\section{Conclusions}

Social function and interaction may be necessary for the motor recovery of stroke patients. Therefore, treatment for enhancing social capabilities or skills to interact with others, and the provision of excellent social support from family can be necessary for functional motor recovery after stroke.

\section{Author contributions}

MC, DW, and BJ designed the research study. SW performed the research. MC and BJ provided help and advice on the neuropsychological evaluations. DW and SW analyzed the data. MC, DW, and BJ wrote the manuscript. All authors contributed to editorial changes in the manuscript. All authors read and approved the final manuscript.

\section{Ethics approval and consent to participate}

The institutional review board approved this study of our hospital (DEF20ORIO079). All procedures performed in studies involving human participants were in accordance with the ethical standards of the institutional and/or national research committee and with the 1964 Helsinki declaration and its later amendments or comparable ethical standards.

\section{Acknowledgments}

We thank the five anonymous reviewers for their helpful suggestions. The present study was supported by a National Research Foundation of Korea grant funded by the Korean government (grant no. NRF-2019R1F1A1061348).

\section{Conflict of Interest}

The authors declare no competing interests.

Submitted: June 02, 2020

Revised: August 26, 2020

Accepted: August 28, 2020

Published: September 30, 2020

\section{References}

Alawieh, A., Zhao, J. and Feng, W. (2018) Factors affecting post-stroke motor recovery: Implications on neurotherapy after brain injury. Behavioural Brain Research 340, 94-101.

Beck, A. T., Epstein, N., Brown, G. and Steer, R. A. (1988) An inventory for measuring clinical anxiety: Psychometric properties. Journal of Consulting and Clinical Psychology 56, 893-897.

Beck, A. T. (1961) An inventory for measuring depression. Archives of General Psychiatry 4, 561.

Chollet, F., Tardy, J., Albucher, J., Thalamas, C., Berard, E., Lamy, C., Bejot, Y., Deltour, S., Jaillard, A., Niclot, P., Guillon, B., Moulin, T.,
Marque, P., Pariente, J., Arnaud, C. and Loubinoux, I. (2011) Fluoxetine for motor recovery after acute ischaemic stroke (FLAME): A randomised placebo-controlled trial. The Lancet Neurology 10, 123-130.

Cummings, J. L. (1997) The Neuropsychiatric Inventory: Assessing psychopathology in dementia patients. Neurology 48, 10S-16S.

D'Anci, K. E., Uhl, S., Oristaglio, J., Sullivan, N. and Tsou, A. Y. (2019) Treatments for poststroke motor deficits and mood disorders: A systematic review for the 2019 U.S. Department of Veterans Affairs and U.S. Department of Defense Guidelines for stroke rehabilitation. Annals of Internal Medicine 171, 906.

Dhand, A., Lang, C. E., Luke, D. A., Kim, A., Li, K., McCafferty, L., Mu, Y., Rosner, B., Feske, S. K. and Lee, J. (2019) Social network mapping and functional recovery within 6 months of ischemic stroke. Neurorehabilitation and Neural Repair 33, 922-932.

Drozdick, L. W., Raiford, S. E., Wahlstrom, D. and Weiss, L. G. (2018) The Wechsler Adult Intelligence Scale-Fourth Edition and the Wechsler Memory Scale-Fourth Edition. In, Drozdick, L. W., Raiford, S. E., Wahlstrom, D. and Weiss, L. G. (eds.) Contemporary intellectual assessment: Theories, tests, and issues, 4th ed. (pp. 486-511). New York, NY, US: The Guilford Press.

Duff, K. (2012) Evidence-based indicators of neuropsychological change in the individual patient: Relevant concepts and methods. Archives of Clinical Neuropsychology 27, 248-261.

Ginex, V., Veronelli, L., Vanacore, N., Lacorte, E., Monti, A. and Corbo, M. (2017) Motor recovery in post-stroke patients with aphasia: The role of specific linguistic abilities. Topics in Stroke Rehabilitation 24, 428-434.

Glass, T. A., Matchar, D. B., Belyea, M. and Feussner, J. R. (1993) Impact of social support on outcome in first stroke. Stroke 24, 64-70.

Glymour, M. M., Weuve, J., Fay, M. E., Glass, T. and Berkman, L. F. (2008) Social ties and cognitive recovery after stroke: Does social integration promote cognitive resilience? Neuroepidemiology 31, 10-20.

Kapur, N. (2005) Neuropsychological Assessment, fourth edition. Journal of Neurology 252, 1290-1291.

Karelina, K., Norman, G. J., Zhang, N. and DeVries, A. C. (2009) Social contact influences histological and behavioral outcomes following cerebral ischemia. Experimental Neurology 220, 276-282.

Kim, H. and Na, D. L. (1999) Normative data on the Korean version of the Boston Naming Test. Journal of Clinical and Experimental Neuropsychology 21, 127-133.

Kim, J. Y., Seo, J. P. and Chang, M. C. (2020) Ataxic hemiparesis after corona radiata infarct: Diffusion tensor imaging correlation of corticoponto-cerebellar tract injury. Translational Neuroscience 11, 13.

Lee, S. Y., Kim, D. Y., Sohn, M. K., Lee, J., Lee, S., Shin, Y., Kim, S., Oh, G., Lee, Y. H., Lee, Y., Joo, M. C., Lee, S. Y., Ahn, J., Chang, W. H., Choi, J. Y., Kang, S. H., Kim, I. Y., Han, J. and Kim, Y. (2020) Determining the cut-off score for the Modified Barthel Index and the Modified Rankin Scale for assessment of functional independence and residual disability after stroke. Plos One 15, e0226324.

McDonald, M. W., Black, S. E., Copland, D. A., Corbett, D., Dijkhuizen, R. M., Farr, T. D., Jeffers, M. S., Kalaria, R. N., Karayanidis, F., Leff, A. P., Nithianantharajah, J., Pendlebury, S., Quinn, T. J., Clarkson, A. N. and O'Sullivan, M. J. (2019) Cognition in stroke rehabilitation and recovery research: Consensus-based core recommendations from the second Stroke Recovery and Rehabilitation Roundtable. International Journal of Stroke 14, 774-782.

Mead, G. E., Legg, L., Tilney, R., Hsieh, C. F., Wu, S., Lundström, E., Rudberg, A. S., Kutlubaev, M., Dennis, M. S., Soleimani, B., Barugh, A., Hackett, M. L. and Hankey, G. J. (2020) Fluoxetine for stroke re- 
covery: Meta-analysis of randomized controlled trials. International Journal of Stroke 15, 365-376.

Moon, J. S., Chung, S. M., Jang, S. H., Won, K. C. and Chang, M. C. (2019) Effects of diabetes on motor recovery after cerebral infarct: A diffusion tensor imaging study. The Journal of Clinical Endocrinology and Metabolism 104, 3851-3858.

Mullick, A. A., Subramanian, S. K. and Levin, M. F. (2015) Emerging evidence of the association between cognitive deficits and arm motor recovery after stroke: A meta-analysis. Restorative Neurology and Neuroscience 33, 389-403.

Nannetti, L., Paci, M., Pasquini, J., Lombardi, B. and Taiti, P. G. (2005) Motor and functional recovery in patients with post-stroke depression. Disability and Rehabilitation 27, 170-175.

Ones, K., Yilmaz, E., Cetinkaya, B. and Caglar, N. (2005) Quality of life for patients poststroke and the factors affecting it. Journal of Stroke and Cerebrovascular Diseases 14, 261-266.

Parikh, R. M., Robinson, R. G., Lipsey, J. R., Starkstein, S. E., Fedoroff, J. P. and Price, T. R. (1990) The impact of poststroke depression on recovery in activities of daily living over a 2-year follow-up. Archives of Neurology 47, 785-789.

Pohjasvaara, T., Vataja, R., Leppävuori, A., Kaste, M. and Erkinjuntti, T. (2001) Suicidal ideas in stroke patients 3 and 15 months after stroke. Cerebrovascular Diseases 12, 21-26.

Povroznik, J. M., Ozga, J. E., Haar, C. V. and Engler-Chiurazzi, E. B. (2018) Executive (dys) function after stroke. Behavioural Pharmacology 29, 638-653.

Reisberg, B., Ferris, S. H., de Leon, M. J. and Crook, T. (1982) The Global Deterioration Scale for assessment of primary degenerative dementia. The American Journal of Psychiatry 139, 1136-1139.

Robinson, R. G., Murata, Y. and Shimoda, K. (1999) Dimensions of social impairment and their effect on depression and recovery following stroke. International Psychogeriatrics 11, 375-384.

Rubio Ballester, B., Bermúdez i Badia, S. and Verschure, P. F. M. J. (2012) Including social interaction in stroke vr-based motor rehabilitation en- hances performance: a pilot study. Presence: Teleoperators and Virtual Environments 21, 490-501.

Shah, S., Vanclay, F. and Cooper, B. (1989) Improving the sensitivity of the Barthel Index for stroke rehabilitation. Journal of Clinical Epidemiology 42, 703-709.

Sparrow, S. S., Cicchetti, D. and Balla, D. A. (2005). Vineland Adaptive Behavior Scales, Second Edition. APA PsycTests.

Tombaugh, T. N. and McIntyre, N. J. (1992) The Mini-Mental State Examination: A comprehensive review. Journal of the American Geriatrics Society 40, 922-935.

Tyburski, E. M., Mak, M., Kurpisz, J., Samochowiec, A. and Potemkowski, A. (2018) Executive dysfunction after stroke - possibilities and limitations of diagnosis. Postepy Psychiatrii $i$ Neurologii 27, 135-145. (In Polish)

Venna, V. R., Xu, Y., Doran, S. J., Patrizz, A. and McCullough, L. D. (2014) Social interaction plays a critical role in neurogenesis and recovery after stroke. Translational Psychiatry 4, e351-e351.

Verhoeven, C. L. M., Post, M. W. M., Schiemanck, S. K., van Zandvoort, M. J. E., Vrancken, P. H. and van Heugten, C. M. (2011) Is cognitive functioning 1 year poststroke related to quality of life domain?. Journal of Stroke and Cerebrovascular Diseases 20, 450-458.

Wechsler, D. (2012) Wechsler Adult Intelligence Scale-Fourth Edition. Pearson.

Wilde, N. J., Strauss, E. and Tulsky, D. S. (2004) Memory span on the Wechsler scales. Journal of Clinical and Experimental Neuropsychology 26, 539-549.

Yi, Z. M., Liu, F. and Zhai, S. D. (2010) Fluoxetine for the prophylaxis of poststroke depression in patients with stroke: A meta-analysis. International Journal of Clinical Practice 64, 1310-1317.

Žikić, T. R., Divjak, I., Jovićević, M., Semnic, M., Slankamenac, P., Žarkov, M. and Žikić, M. (2015) The effect of post stroke depression on functional outcome and quality of life. Acta Clinica Croatica $\mathbf{5 3}$, 294-301. 